\title{
marges Marges
}

revue d'art contemporain Revue d'art contemporain

L'art avec (ou sans) le marché de l'art

\section{«Photographie, arme de classe »}

Paris, Centre Pompidou, Galerie de photographies - 7 novembre 2018 4 février 2019

Fedora Parkmann

\section{(2) OpenEdition}

1 Journals

Édition électronique

URL : https://journals.openedition.org/marges/1891

DOI : $10.4000 /$ marges. 1891

ISSN : 2416-8742

Éditeur

Presses universitaires de Vincennes

\section{Édition imprimée}

Date de publication : 19 avril 2019

Pagination : 142-143

ISBN : 978-2-84292-996-1

ISSN : 1767-7114

Référence électronique

Fedora Parkmann, « «Photographie, arme de classe» », Marges [En ligne], 28 | 2019, mis en ligne le 19 avril 2019, consulté le 07 janvier 2022. URL : http://journals.openedition.org/marges/1891 ; DOI :

https://doi.org/10.4000/marges.1891 


\section{«Photographie, arme de classe »}

\section{Paris, Centre Pompidou, Galerie de photographies}

7 novembre 2018 - 4 février 2019

Cette exposition et son catalogue dirigés par Damarice Amao, Florian Ebner et Christian Joschke constituent l'aboutissement très attendu d'un projet de recherche qui s'est attaqué pendant près de quatre ans à un pan méconnu de l'histoire de la photographie de l'entre-deux-guerres en France : la fabrication, entre 1928 et 1936, d'une culture visuelle de gauche, grâce à la collaboration de photographes modernes et de photoamateurs ouvriers affiliés à des associations culturelles communistes. On ne savait pas grand-chose des acteurs, des modes d'organisation et des canaux de diffusion de cette production engagée, par manque de sources - beaucoup restent d'ailleurs encore à découvrir, de l'aveu même des commissaires, notamment les photographies anonymes produites par les amateurs ouvriers. Ouvrir le chantier français s'avérait d'autant plus indispensable que la photographie sociale et documentaire de l'entre-deux-guerres rencontre l'intérêt des chercheurs, comme l'a montré la dernière synthèse internationale de la question, l'exposition "The WorkerPhotography Movement» qui s'est tenue à Madrid en 2011. Les récentes expositions monographiques consacrées quant à elles à des figures bien connues de l'engagement - Henri Cartier-Bresson au Centre Pompidou en 2014 et Eli Lotar au Jeu de Paume en 2017 - demandaient à être complétées par une analyse portant sur les pratiques collectives de la photographie engagée, tâche à laquelle s'attèle la présente manifestation. Répartie en sept sections thématiques, elle éclaire d'abord les supports de diffusion de cette imagerie - l'exposition et l'illustré - avant d'en décliner les différents thèmes iconographiques, du pittoresque social aux conflits étrangers, en passant par l'éden ouvrier, la photographie qui accuse et les mobilisations. Malgré son approche transversale, la démonstration n'évacue pas pour autant la figure du photographe auteur; les commissaires ont en effet abondamment puisé à la collection de Christian Bouqueret, essentiellement composée de tirages d'auteur, qui a été acquise par le Musée national d'art moderne en 2011. De ce fait, une place conséquente est allouée aux personnalités phares de la modernité photographique qui ont rejoint l'association des 
écrivains et artistes révolutionnaires (AEAR) et traité de thématiques sociales: Henri Cartier-Bresson, Germaine Krull, Eli Lotar, Willy Ronis et quelques autres. À première vue, la préférence semble donc avoir été donnée aux belles épreuves d'exposition de ces photographes confirmés, au détriment de la production de la base militante - un choix qui s'explique par la nature même des collections du musée. Ce déséquilibre est toutefois compensé par les épreuves anonymes réparties de façon égale dans les sections thématiques du parcours d'exposition. Des opérateurs moins connus - photo-amateurs de l'ombre ou personnalités périphériques - sont par ailleurs rappelés à la mémoire, du moins en images, car le catalogue ne fournit pratiquement pas d'informations biographiques à leur sujet. Enfin et surtout, la photographie imprimée sous toutes ses formes - presse illustrée, affiche ou tract - occupe une place à part entière dans les vitrines et même sur les cimaises, où des pages de magazine se superposent aux photographies encadrées. En cohérence avec la logique documentaire revendiquée par les photographes eux-mêmes, ce judicieux choix d'accrochage revient à signifier que le tirage argentique n'avait pas plus de valeur que la reproduction photomécanique; seule comptait leur capacité à attester d'une situation sociale.

Car ce qui prime ici sur l'approche biographique et l'analyse formelle, c'est l'économie de cette pratique engagée et collective de la photographie, la manière dont s'organisent la production et la diffusion des images. L'exposition montre, au moyen de documents d'archives, que le photographe - qu'il soit professionnel, artiste ou ouvrier - se mobilise au sein de l'association dont il est membre, laquelle le relie au parti communiste et à ses organes de presse, puis par capillarité, à tout un réseau transnational orchestré par le Komintern. Ce contexte international est suggéré par le diagramme figurant à l'entrée de l'exposition, les rapprochements entre presse illustrée française, allemande et soviétique opérés dans l'accrochage ainsi que par la section thématique "Théâtres extérieurs du conflit social ». Entre l'individu et le collectif, entre le local et le global, l'image circule, change de forme et intervient dans l'espace public, là où se jouent les luttes politiques. "Le politique ne s'écrit pas seulement dans le message véhiculé par l'image mais aussi dans l'économie même du médium photographique ", avancent les commissaires dans l'introduction au catalogue. Par rapport aux lectures traditionnelles de l'art engagé, la logique est donc inversée: c'est moins la politique qui détermine l'aspect des images et leur message, que l'image dans sa matérialité - qu'elle soit photographie, cliché ou reproduction - qui rejoint le champ des luttes et infléchit le cours de la politique elle-même. Afin de mieux éclairer la manière dont les images s'articulent avec l'engagement politique, les commissaires ont choisi d'interroger aussi bien les formes et les discours que les conditions de production et les usages, en accord avec le tournant matériel pris par la recherche en photographie ces dernières années.

Ce que l'exposition et son catalogue dévoilent de la structure du mouvement français et de l'économie de l'image engagée apparaît suffisamment organique pour être applicable à d'autres contextes où s'est épanouie la photographie sociale. En mettant ainsi en lumière, avec une grande intelligence, l'un des faisceaux de cette production transnationale, elle encourage des recherches nouvelles qui viendraient enrichir à leur tour la prise de vue globale.

\section{Fedora Parkmann}

